\title{
La Metrópoli del Valle de Toluca y sus Retos hacia la Sustentabilidad
}

\author{
The Metropolis of the Toluca Valley and its Challenges towards \\ Sustainability
}

\section{A Metrópole do Vale do Toluca e seus Desafios para a Sustentabilidade}

J. Loreto Salvador Benítez

Doctor en Humanidades: Ética

Universidad Autónoma del Estado de México

jlsalvadorb@uaemex.mx

(iD) https://orcid.org/0000-0003-3438-9539

Leonardo Alberto Torres González

Arquitecto, MSc (c) Estudios Sustentables, Regionales y Metropolitianos

Universidad Autónoma del Estado de México

leonardo.torres@gmail.com

(iD) https://orcid.org/0000-0001-9109-8135

Recibido: diciembre 16 de 2021 Aceptado: febrero 15 de 2022 Publicado: febrero 16 de 2022

\section{RESUMEN}

El objetivo de este trabajo es analizar el caso de estudio, Zona Metropolitana del Valle de Toluca, desde el desarrollo sostenible; su vertiginoso crecimiento e impacto de dos megaproyectos plantean problemáticas debido a las insuficientes políticas públicas que regulen su desarrollo en infraestructura. Se trata de un estudio documental y cualitativo, de participación-acción, que permite prever consecuencias diversas en una metrópoli de alta concentración humana y limitadas acciones de coordinación intermunicipal. Se precisa asumir responsabilidad social, empresarial, institucional y gubernamental, para afrontar dilemas y disyuntivas urbanas en el sentido de la Agenda 2030 y de la ciudad compleja.

Palabras clave: Sustentabilidad; Complejidad; Zona Metropolitana; Valle de Toluca.

\section{ABSTRACT}

The objective of this work is to analyze the case study, Toluca Valley Metropolitan Area, from a sustainable development perspective; its dizzying growth and impact of two megaprojects pose problems due to insufficient public policies that regulate its development in infrastructure. It is a documentary and qualitative study of participation-action, which allows to foresee various consequences in a metropolis with high human concentration and limited inter-municipal coordination actions. It is necessary to assume social, business, institutional and governmental responsibility, to face urban dilemmas and dilemmas in the sense of the 2030 Agenda and of the complex city.

Keywords: Sustainability; Complexity; Metropolitan area; Toluca Valley. 


\section{RESUMO}

O objetivo deste trabalho é analisar o estudo de caso, Área Metropolitana do Vale de Toluca, a partir de uma perspectiva de desenvolvimento sustentável; seu vertiginoso crescimento e impacto de dois megaprojetos colocam problemas devido à insuficiência de políticas públicas que regulam seu desenvolvimento em infraestrutura. Trata-se de um estudo documental e qualitativo de participação-ação, que permite antever várias consequências numa metrópole com elevada concentração humana e limitadas ações de coordenação intermunicipal; É preciso assumir responsabilidade social, empresarial, institucional e governamental, para enfrentar dilemas e dilemas urbanos no sentido da Agenda 2030 e da cidade complexa.

Palavras-chave: Sustentabilidade; Complexidade; Área metropolitana; Vale de Toluca.

\section{INTRODUCCIÓN}

El desarrollo sustentable, la protección del medio ambiente y la modernización de las ciudades son factores que, si bien llevan años siendo prioritarios en países con un desarrollo y capitalización importante, como es el caso de los países europeos, en América Latina apenas comienza a formar parte de las agendas políticas.

Sin embargo, comienza a notarse el hecho de que no es posible seguir al pie de la letra las estrategias de los países considerados de primer mundo: las características regionales y económicas son muy particulares, los países latinoamericanos están en pleno desarrollo o en situación de pobreza, razón por la cual existe la necesidad de implementar políticas ecológicas específicas, enfocadas en resolver estos problemas desde un punto de vista local.

El presente estudio se realizó bajo la experiencia previa que se obtuvo trabajando en la administración municipal del Ayuntamiento de Toluca (2016-2018), en donde se identificaron las facultades que tiene la misma en la implementación de acciones desde este nivel de Gobierno; asimismo, se detectaron los puntos débiles que impiden a las municipalidades poder asumir una injerencia de mayor alcance dentro de sus propias ciudades, y la ausencia de una comunicación y coordinación intermunicipal, que favorezca el intercambio de experiencias y estrategias, desde los planes de desarrollo municipal, para atender y resolver problemáticas de un todo como conjunto, de la denominada Zona Metropolitana del Valle de Toluca-Lerma (ZMVT).

Asimismo, mediante el uso de los lineamientos de la Agenda 2030 como guía se contraponen las necesidades de la Metrópoli con las actuales acciones y políticas públicas existentes.

\section{METODOLOGÍA}

Este trabajo corresponde al avance de un proyecto de investigación en el marco de la Maestría en Estudios Sustentables, Regionalesy Metropolitanos, de la Universidad Autónoma del Estado de México. La ciudad de Toluca, capital de uno de los estados fuertes, económica, demográfica y políticamente de México, es el objeto de estudio por los cambios vertiginosos que tienen lugar, pasando de ser provincia a una urbe que comprende diversas municipalidades. El fenómeno de metropolización se ha observado como agentes-ciudadanos que somos, testigos y partícipes del crecimiento, en apariencia ordenado, pero donde es posible identificar asimetrías y ausencias de coordinación gubernamental. Se trata de un estudio documental, cualitativo, de participación-acción desde el interior de la administración pública del Ayuntamiento de Toluca, como del crecimiento urbanístico, donde es posible identificar megaproyectos de gran impacto medioambiental, como el tren interurbano que conectará a la ZMVT con la ciudad de México (CDMX), y el Parque de los Fundadores recién inaugurado en el centro histórico de Toluca. Exponemos dichos proyectos como sus probables impactos en la perspectiva de la sustentabilidad. Se parte de un marco teórico-conceptual en que se fundamenta el caso de estudio, donde destacan la sustentabilidad, la metrópoli y la responsabilidad, que permite exponer la problemática y trazar prospectivas desde la participación ciudadana.

\section{La sustentabilidad en México}

Para que México logre adoptar de manera eficiente el desarrollo sostenible, debemos atender la ecología política desde una visión global que aborde aspectos sociales, económicos y naturales, sin menoscabo del cimiento cultural. Solamente 
al concientizar esta relación compleja podremos facilitar la inserción de estas ideas dentro de las poblaciones y la integración de políticas públicas claras que promuevan, faciliten y sancionen en el contexto del ser social.

México tiene una tarea difícil; a diferencia de los países Europeos que abordan la sostenibilidad desde cimientos de desarrollo firmes; nuestro país deberá atender ambas necesidades a la par, mantener un ideal de progreso y competitividad global y a su vez implementar políticas sustentables.

Para lograr este proceso de sustentabilidad en México debemos tomar en cuenta el concepto de Ciudad Saludable, que según Hancock la cual se define como: "Ciudad que crea y mejora constantemente su entorno físico y social, además de ampliar los recursos comunitarios para que las personas puedan apoyarse unos a otros al realizar todas las funciones de la vida y desarrollar su potencial máximo" (Estrada, Aguero y Cuenca, 2013, p.48).

La noción sustentable involucra varias dimensiones que tienen que ver con los bienes y recursos naturales, en su relación con el metabolismo que se establece entre los seres vivos con la Madre Tierra. Si bien, la ciencia nos muestra una realidad fragmentaria desde las diversas disciplinas, lo que ha permitido comprender el fenómeno de la vida como una función producto de la evolución; lo cierto es que desde la perspectiva de la complejidad, los entes bióticos y abióticos se vinculan íntimamente en una red de interrelaciones. Interesa explicar y comprender la idea de complejidad, en este sentido, en una entidad que es muestra fehaciente de una red de interrelaciones que dan funcionalidad y sentido: la Ciudad.

Para abordarel tema de sostenibilidad en un territorio debemos tomar en cuenta todos los factores que intervienen en el objeto de estudio, es el caso que se analizará: la Zona Metropolitana del Valle de Toluca (ZMVT). Las ciudades y principalmente las metrópolis son sistemas complejos, en las que sus elementos están interdefinidos. Es decir, que para estudiarlos debemos atender sus interconexiones.

Pero ¿qué entender por complejidad? Se trata de un fenómeno cuantitativo, "una cantidad extrema de interacciones e interferencias entre un número muy grande de unidades" (Morín, 2003, p.105). Todo sistema viviente, autoorganizado, el más básico, combina enorme número de unidades que desafían al cálculo elemental; pero lo que importa es la incertidumbre que implica lo aleatorio y la indeterminación; dicho de otra manera, la complejidad se relaciona con el azar (Morin, 2003, p.50). Esta noción importa al dar un paso más allá de las disciplinas, y con ello en el reduccionismo de la especialización; pues la crítica a esta es la disolución que realiza del objeto de estudio, por ejemplo, la ciudad, la naturaleza, el mismo ser humano.

Esta categoría posibilita entonces nuevos abordajes a problemáticas diversas; la sociedad como la naturaleza pueden ser comprendidas mucho mejor desde esta perspectiva epistemológica, teórica y metodológica. La metrópoli como fenómeno planeado o derivado de un crecimiento exorbitante, incierto, puede estudiarse desde la noción reciente de "ciudades complejas"; pero lo mismo aplica para la periferia y las regiones, que permiten explicar y comprender la relación dialéctica entre la parte y el todo, el centro y la periferia.

Así, una región está conformada por sus procesos territoriales, su organización político-administrativa, espacios públicos, arquitectura, economía $y$, por supuesto, por sus habitantes, sin embargo, las características propias de cada ciudad se dan por las relaciones que existen entre cada uno de estos elementos. Hay una correlación estrecha, e indisoluble, entre el centro-ciudad y la periferiaregión(es).

Cabrera $(2013$, p.18) señala que, la concepción de complejidad es una necesidad insustituible para la resolución de problemáticas de las ciudades latinoamericanas, ciudades que tienden cada vez más a la Metropolización; también precisa que las estrategias de acción dirigidas a las metrópolis están en sus inicios.

Las metrópolis configuran ejemplos complejos de la fusión territorial y funcional de varios municipios, configurando sistemas administrativos, normativos y funcionales en el marco de políticas públicas, federales, estales y municipales. Abordar estos procesos para su estudio, debería tomar en cuenta la funcionalidad vertical y transversal de las ciudades que funcionan como Unidad espacioregional, y que, desde una perspectiva optimista generan oportunidades de trabajo, en el desarrollo individual de quienes habitan la metrópoli. 


\section{El Valle de Toluca como Metrópoli}

Toluca, la capital del Estado de México posee una de las zonas industriales más importantes del país, además de estar geográficamente muy cerca de la Ciudad de México lo cual genera fenómenos de movilidad entre estos dos espacios a una gran escala; así mismo, este municipio es también sede de la Universidad Autónoma del Estado de México, UAEMex, factor que genera una migración interna del Estado y del país.

El acelerado proceso de crecimiento de este Municipio provoca que hacia 1994 se considerara la primera visión de metrópoli de este espacio geográfico, según afirma Estrada et al. (2013, p.113); con fines económicos y con base en las actividades de los municipios se plantean, por parte del Gobierno del Estado de México, dos nuevas metrópolis, la Cuautitlán-Texcoco y Toluca, la cual se formaba en conjunto con los territorios pertenecientes a los municipios de Metepec, Lerma, San Mateo Atenco y Zinacantepec; este planteamiento es propuesto con la idea de facilitar el desarrollo de las actividades económicas, pero que nacen de la urbanización producto de la intensa migración del país.

De acuerdo con Santana, Rosales, Manzano, Santana y Pineda (2013, p.15), hacia el año 2012 la ZMVT estaba conformada según datos del INEGI por 15 Municipios: Almoloya de Juárez, Calimaya, Chapultepec, Lerma, Metepec, Mexicaltzingo, Ocoyoacac, Otzolotepec, Rayón, San Antonio la Isla,
San Mateo Atenco, Temoaya, Toluca, Xonacatlán y Zinacantepec.

En la Gaceta de Gobierno del Estado de México se aprueba en la administración 2009-2015, la Declaratoria de Zona Metropolitana del Valle de con 22 Municipios (IPOMEX, 2015).

En la figura 1 se aprecian las municipalidades, y su respectiva diferenciación como zonas; la de Toluca y la de Santiago Tianguistengo que, incluso esta última, colinda con las montañas de la sierra del Ajusco, limítrofe con la Ciudad de México y el estado de Morelos.

El crecimiento demográfico de los últimos 30 años, en el también llamado valle de los matlazincas, ha sido exponencial y ha obligado a estas transformaciones territoriales; tanto ha sido el crecimiento de la ZMVT, que según Santana, Rosales, Manzano, Santana y Pineda (2013, p.81), el Gobierno del Estado reconoce que en el Valle de Toluca se genera un nuevo caso de estudio y de preocupación, ya que la unión de la Zona Metropolitana del Valle de Toluca en conjunto con la Zona Metropolitana del Valle de México, están generando una nueva Metrópoli por sí misma. Se trata de un fenómeno donde la ciudad-centro de infraestructura urbana, va devorando paulatinamente zonas de cultivo de comunidades y pueblos que la circundan. Ello ocurrió así en la metrópoli de la Ciudad de México durante los últimos cincuenta años, cuya mancha urbana absorbió territorios cultivables; y lo mismo está ocurriendo ahora en la ZMVT (Tabla 1).

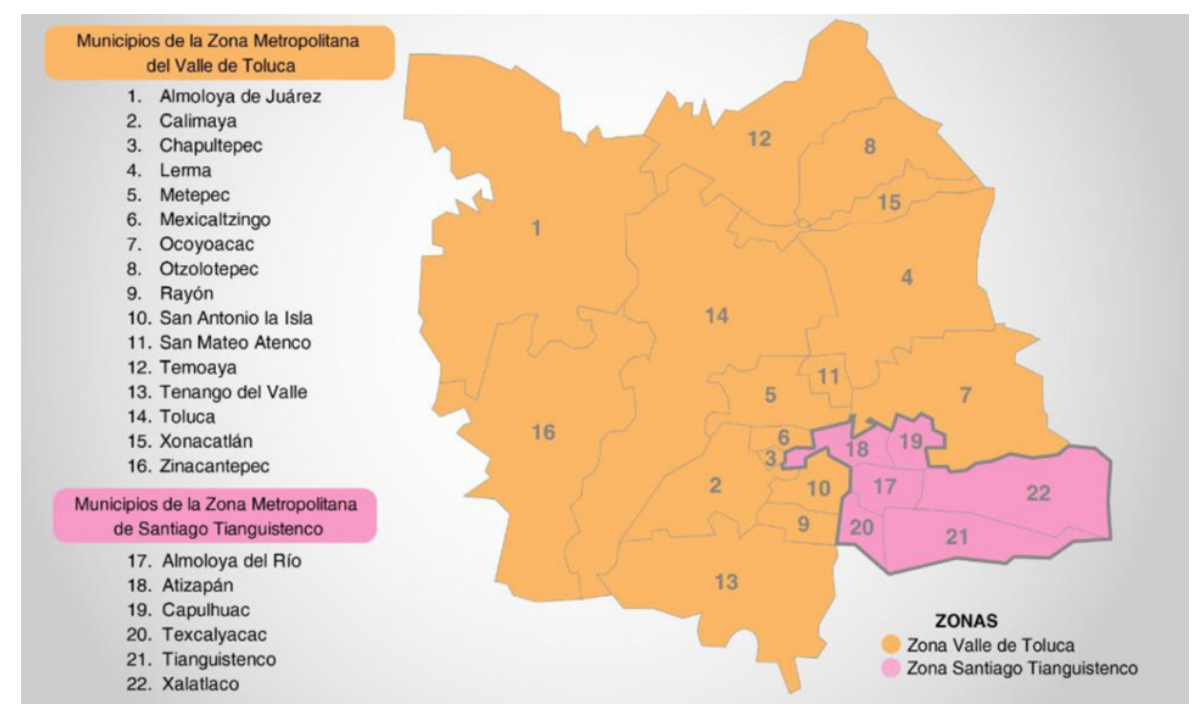

Figura 1: Mapa de la Zona Metropolitana del Valle de Toluca. Fuente: Página del Gobierno del Estado de México (2021) 
Tabla 1: Crecimiento poblacional en las Zonas Metropolitanas del Valle de Toluca y Conurbada

\begin{tabular}{|c|c|c|c|c|c|c|c|c|c|}
\hline & \multirow{2}{*}{$\begin{array}{c}\text { PAIS } \\
\text { POBLACION }\end{array}$} & \multirow{2}{*}{$\begin{array}{c}\begin{array}{c}\text { ESTADO DE } \\
\text { MÉXICO }\end{array} \\
\text { POBLACION }\end{array}$} & \multicolumn{3}{|c|}{$\begin{array}{c}\text { ZONA } \\
\text { METROPOLITANA CONURBADA }\end{array}$} & \multicolumn{3}{|c|}{ REGION DEL VALLE DE TOLUCA (RVT) } \\
\hline \multirow{9}{*}{ POBLACIÓN } & ANO & & & POBLACION & $\begin{array}{l}\text { PROPORCION } \\
\text { ESTADO \% }\end{array}$ & \begin{tabular}{|c|} 
DENSIDAD \\
POBLACIONAL \\
HAB / KM2 \\
\end{tabular} & POBLACION & $\begin{array}{l}\text { PROPORCION } \\
\text { ESTADO \% }\end{array}$ & $\begin{array}{l}\text { DENSIDAD } \\
\text { POBLACIONA } \\
\text { L HAB / KM2 } \\
\end{array}$ \\
\hline & 1960 & 34,923 & 1,898 & 176 & 9.27 & 179.09 & 437 & 23.02 & 163.98 \\
\hline & 1970 & 48,225 & 3,833 & 270 & 7.04 & 261.19 & 617 & 16.10 & 231.29 \\
\hline & 1980 & 66,864 & 7,564 & 500 & 6.61 & 405.77 & 939 & 12.41 & 351.66 \\
\hline & 1990 & 81,249 & 9,815 & 886 & 9.03 & 555.33 & 1,249 & 12.73 & 467.93 \\
\hline & 1995 & 91,158 & 11,708 & 1,159 & 9.90 & 664.37 & 1,501 & 12.82 & 562.30 \\
\hline & 2000 & 97,015 & 13,097 & 1,401 & 10.70 & 770.13 & 1,733 & 13.23 & 649.11 \\
\hline & 2010 & 112,231 & 15,592 & 1,718 & 11.02 & & 2,124 & 13.62 & \\
\hline & 2020 & 122,107 & 17,627 & 1,858 & 10.54 & & 2,296 & 13.03 & \\
\hline \multirow{7}{*}{$\begin{array}{l}\text { TASA DE } \\
\text { CRECIMIENT } \\
\text { O MEDIA } \\
\text { ANUAL }\end{array}$} & $1960-1970$ & 3.40 & 7.56 & 3.99 & & & 3.63 & & \\
\hline & $1970-1980$ & 3.20 & 6.78 & 4.35 & & & 4.13 & & \\
\hline & $1980-1990$ & 2.02 & 2.07 & 3.26 & & & 2.97 & & \\
\hline & 1990-1995 & 2.06 & 3.17 & 3.22 & & & 3.30 & & \\
\hline & $1995-2000$ & 1.58 & 2.65 & 3.51 & & & 3.41 & & \\
\hline & $2000-2010$ & 1.47 & 1.76 & 1.69 & & & 1.76 & & \\
\hline & $2010-2015$ & 0.85 & 1.23 & 1.27 & & & 1.27 & & \\
\hline
\end{tabular}

Fuente: Gobierno del Estado de México; Secretaría de Desarrollo Urbano y Vivienda, Datos 1960-2000 fuente de información Censos Generales de Población y Vivienda del Estado de México 1960, 1970, 1980, 1990 y 2000 y conteo 1995 INEGI

Con datos como este, se ve la pertinencia de analizar los efectos adversos que el nacimiento de una Megametrópoli puede ocasionar a los Municipios del Valle de Toluca, que si bien, como hemos visto, ha tenido un crecimiento demográfico y desarrollo acelerado, no se compara con el que se vive dentro del Valle de México; en este sentido, proyectos como el nuevo Tren Interurbano deben ser tomados en cuenta, no solo por los efectos y estragos naturales que se generan en espacios como terminales y zonas de transporte, sino porque este proyecto podría, en gran medida, afianzar o finalizar este proceso de unificación de dos grandes Metrópolis.

De acuerdo con Cabrera (2013, p.31), uno de los principales problemas de las Metrópolis es la segregación de las zonas rurales o menos competitivas, es decir, cuando varios municipios se unen, los centros de producción y consumo, como es el caso de Toluca y Metepec, tienden a un acelerado crecimiento económico; sin embargo, las periferias de las metrópolis en contraste, tienden a ser zonas de pobreza y rezago. De Mattos (1999), hablaba de un crecimiento desigual y divergente, producto justamente de este crecimiento endógeno por parte de los municipios "Corazón" de las Metrópolis.

La pobreza y el rezago social no son el único problema que nace de las Metrópolis; las afectaciones al medio ambiente también son graves, el acelerado crecimiento demográfico y de asentamientos humanos carentes de una planeación urbana, generan la desaparición de espacios de cultivo o incluso de áreas boscosas, tal es el caso de zonas como Cacalomacán y Santa Cruz Cuahutenco, las cuales tienen a la venta cientos de hectáreas ejidales, con uso agrícola, en donde comienza a percibirse un crecimiento poblacional por la falta de espacios habitacionales dentro de la ciudad.

Otro caso es la zona de Calimaya que desde hace algunos años es centro de desarrollos habitacionales horizontales a gran escala, con un mercado de interés medio alto, pero que ha destruido grandes espacios naturales.

En este sentido habitacional las Metrópolis acarrean como define Cabrera (2013, p.18), problemas "Duales" en los que la demanda de espacios habitacionales es alta por la migración externa y por los habitantes de zonas rurales que se ven en la necesidad de trasladarse a las ciudades;

\section{Problemáticas de la ZMVT}

Toluca, la Capital del Estado de México y corazón de la Metrópoli, constituye un reto para el Ayuntamiento (2022-2024). El desarrollo y crecimiento económico, social y de infraestructura es inminente con la entrada en operación del Tren Interurbano, que conectará a los dos grandes valles denominados Altiplano mexicano; y el Parque Fundadores en el Centro Histórico; proyectos que, sin lugar a dudas, traerán consigo beneficios económicos y sociales 
a la ciudad y a la Región centro del país, pero a su vez, generará problemas de carácter ambiental y social, los cuales se incrementarán a corto mediano y largo plazo.

La construcción del megaproyecto del Tren Interurbano, Toluca-CDMX viene establecido en el Plan Nacional de Desarrollo 2013-2018, y su fecha de entrega se programó al término de esa administración federal; no obstante, por el incremento en los costos la obra no se concluyó como se tenía previsto. El Gobierno entrante retomó dicho proyecto y asume el compromiso de terminarlo en 2022, debido al interés público que representa por la movilidad masiva que tendrá lugar entre las dos metrópolis.

De acuerdo con García (2019, p.109), esta obra cuenta con un sistema moderno, considerado único en su género en Latinoamérica, y que compite con grandes obras similares como las que se encuentran en New York, Madrid, Ámsterdam y Londres; Sin embargo, los daños ambientales que acarrea su construcción son incalculables ya que, según García, su construcción ha devastado Áreas Naturales Protegidas como El Parque Nacional Insurgente "Miguel Hidalgo y Costilla", mejor conocido como "La Marquesa". Además de esto, se prevé que las terminales de este proyecto traerán problemas de tránsito y contaminación, además de inseguridad, la cual no solo será de carácter local, ya que el mismo tren permitirá el rápido acceso de nuevos grupos delictivos de otras zonas del estado y de la Ciudad de México.

Otro megaproyecto es el Parque Fundadores, inaugurado en octubre del 2021, en Toluca; sin lugar a duda un gran avance en cuanto a infraestructura cultural de los últimos 30 años en la Región, que atraerá visitantes de municipios aledaños y probablemente de ciudades cercanas, sin embargo, la capacidad del Centro Histórico en cuanto a vialidades, y estacionamientos no es la adecuada para recibir grandes cantidades de automóviles y visitantes.

Sin duda, la Ciudad de Toluca como Capital del Estado, y como centro gravitacional de la Zona Metropolitana que representa, requiere de estos nuevos espacios y de un desarrollo en materia de infraestructura sustentable, cambios que incluso llegaron de manera tardía y que aun distan de ser adecuados, sin embargo, se cuestiona la capacidad de la ciudad en infraestructura para soportarlo.
El desarrollo de las ciudades y la adopción de nuevas tecnologías e infraestructura sustentable es, a nivel mundial, un requerimiento desde el punto de vista de competencia económica y por supuesto, desde la visión de sostenibilidad, la cual comienza a formar parte de las agendas políticas globales, y una exigencia para lograr alcanzar las metas de los ODS en pro de un futuro digno, pero, ¿están la ciudades latinoamericanas y en caso concreto el área metropolitana de Toluca preparadas para ello?.

El caso de estudio de la Zona Metropolitana del Valle de Toluca (ZMVT) los municipios que la integran tienen la tarea de generar un diálogo intermunicipal constante, el cual hoy en día es casi inexistente; es necesario que administrativa y políticamente, desde los gobiernos municipales, se reconozca el carácter metropolitano de las problemáticas que se viven estructural, demográfica, económica y socialmente.

Es de suma importancia a su vez, que los gobiernos Estatal y Federal apoyen las acciones necesarias para el adecuado y óptimo desarrollo sustentable de la ZMVT, mediante el diseño e implementación de políticas públicas regionales.

\section{Ciudad y Responsabilidad Social}

Como se comentó en el punto anterior la capacidad de la Zona Metropolitana del Valle de Toluca de atender las necesidades en materia de sostenibilidad recae en la organización de todos los actores que la integran, Gobierno, sector empresarial y la ciudadanía, por lo que la responsabilidad social de cada uno de estos sectores es de vital importancia.

La responsabilidad como concepto implica un carácter ético, sin embargo, la responsabilidad no surge solamente desde una visión moral; para Ingarden (1980, p.15) la responsabilidad puede nacer de distintas situaciones, como cuando el individuo tiene responsabilidad de algo o alguien. Cuando uno asume una responsabilidad de algo. Cuando uno es hecho responsable de algo. $\mathrm{O}$ simplemente el obrar responsablemente.

Hablando específicamente del proyecto o de manera global de la idea de sustentabilidad solemos pensar que la responsabilidad que tenemos hacia el ambiente, la sociedad y el futuro se relaciona a lo que Ingarden (1980, p.15) describía 
simplemente como obrar responsablemente, con hacer lo correcto.

Es decir, cuando hablamos de obrar responsablemente, nos referimos a que estamos conscientes de la razón que nos ha llevado a realizar las acciones y que tenemos una intención determinada, como puede ser el apoyo al medio ambiente.

Sin embargo no es así, la responsabilidad se aplica de manera automática a quien realiza una acción y uno se ve automáticamente responsabilizado por las repercusiones y efectos de ello (Ingarden, 1980 p.20), es decir, que las acciones que podamos realizar como ciudadanos o como habitantes de este planeta no son solamente desde un actuar u obrar responsable, sino que estamos haciéndonos conscientes de que las acciones diarias que realizamos, tienen una repercusión en la sociedad que nos rodea, en el ambiente y la economía, por lo que tenemos la tarea de actuar en la mitigación de este actuar, porque somos participes directos del problema.

Asimismo, la participación de la sociedad civil dentro de la toma de decisiones que conciernen a la ciudad es también parte de esta llamada responsabilidad social, como ciudadanos tenemos no solo el derecho, sino la obligación de ser partícipes directos en la construcción y evolución de nuestras ciudades, a esto se le conoce como el derecho a la ciudad.

La responsabilidad social puede también dividirse de acuerdo al sector, ya sea gubernamental, empresarial o universitaria, los cuales analizaremos de manera independiente. Sin embargo, el sentido de responsabilidad comienza desde lo individual, y si bien, como se comentó esto puede tener distintas vertientes, nos enfocaremos en la que atañe a la ciudad y la sustentabilidad.

El derecho a la ciudad se considera un nuevo derecho humano, en el que los ciudadanos sean actores en la transformación de las ciudades modernas, Lefebvre (1991, p.125) señalaba que este derecho incluía no solo la inclusión en la toma de decisiones sino el uso intenso de la ciudad y el uso pleno de sus lugares de encuentro e intercambio social.

Para Borja (2008), los "nuevos derechos urbanos" que contribuyan a la "justicia de la ciudad" como el derecho al lugar, al espacio público, la monumentalidad, la belleza, la identidad colectiva, la movilidad, la accesibilidad, la conversión de la marginalidad urbana en ciudadanía, el Gobierno metropolitano, al acceso y uso de las tecnologías de comunicación e información; es decir, se concibe este derecho como un sistema complejo e interdisciplinario como la ciudad misma.

En este sentido las comunidades que integran la Zona Metropolitana de Toluca, o de cualquier metrópoli, constituyen en sí mismos, un sistema complejo como asegura Cabrera (2013, p.18); y por lo tanto deben ser tratados como tal, desde una visión multidisciplinaria; es ahí en donde remarcamos la importancia de que los ciudadanos desde su individualidad aporten de alguna manera al cambio de su ciudad y de su país; de ahí mismo la importancia de que la participación en las acciones que definan el rumbo de los territorios sea desde visiones tanto personales como empresariales y gubernamentales.

El presente trabajo destaca también la importancia de la responsabilidad social universitaria, porque desde la academia se forman los futuros empresarios, integrantes de la clase políticoadministrativa, ciudadanos, profesionales; es decir, la academia es el punto medular de donde debería nacer cualquier motivación de ejercer ese derecho a la ciudad y esa obligación como ciudadano.

La responsabilidad social es una cuestión que recae no solo en los gobiernos sino en los individuos y en las empresas, un sector que muchas veces tiene más capacidad económica o de acción para incidir en algún cambio positivo para la sociedad. El Valle de Toluca cuenta con una de las zonas empresariales y de producción más importante del país, por lo que es vital su inclusión dentro de las estrategias que se planteen en favor de los ODS.

La actuación de las empresas no debe ser meramente económica, es decir basada en ofrecer productos, bienes y servicios sino que debe de ir más allá, ya que afecta directa o indirectamente a la naturaleza y a la sociedad. Según Serna (2006, p.154) la responsabilidad social empresarial nace en un inicio con cuatro componentes principales: económicos, legales, éticos y filantrópicos, conceptos que pueden dejar muy al aire la respuesta de las empresas frente a los impactos que generan a su alrededor ya que tienen, salvo la parte legal, un carácter opcional y al estar muchas veces en 
contra de la meta final de las corporaciones que es el de producción y acumulación de capital, impide que se desarrollen acciones pertinentes.

Está claro que el componente que debería ser clave para las empresas es la parte ética, ya que el entendimiento de los impactos que generan, ya sean, económicos, sociales o ambientales, les permitiría generar estrategias de mitigación, sin embargo, para las empresas el desarrollo es primordial y es entendible, por lo que al igual que como se ha entendido la necesidad del desarrollo de manera global, con un equilibrio sostenible, las corporaciones tienen este mismo derecho, por lo que las nuevas estrategias se basan más en que su producción tenga un equilibrio con los impactos que generan, es decir, dentro de las empresas también respaldan la sostenibilidad, Elkington (2004, pp.1-3) lo describe como la capacidad de las corporaciones de entregar al mismo tiempo beneficios económicos, sociales y ambientales a los que denomina triple bottom line.

La ONU también se ha comprometido a verificar las acciones de las empresas en pro de la sustentabilidad, por lo que crea un Modelo de Liderazgo en la Sostenibilidad Corporativa (2010, p.4), un programa que permite que las empresas demuestren su grado de compromiso con la sostenibilidad.

Sin embargo, como hemos comentado antes, la responsabilidad debe verse de forma transversal y debemos buscar estrategias que permitan facilitar los procesos a cada uno de los actores; las empresas sin lugar a dudas son quienes mayor impacto tienen hacia el ambiente, pero su producción va ligada a nuestras acciones como consumidores; entonces la responsabilidad no es solo de las empresas hacia sus procesos productivos, sino nuestra, al optar por productos sustentables o disminuir nuestro consumo.

De igual manera, al hablar de transversalidad en las acciones del proyecto, los gobiernos y las empresas lejos de funcionar de manera independiente pueden llegar a acuerdos, que les permitan generar acciones para efectos del proyecto. Quizás el Gobierno no tenga el presupuesto necesario para hacer intervenciones importantes en todos los espacios públicos, pero, ¿qué pasaría si las empresas pudieran «adoptar» espacios? Por ello destacamos la importancia del trabajo conjunto y de políticas que no solo promuevan, sino que incentiven esta transversalidad.

\section{RESULTADOS Y DISCUSIÓN}

Se han abordado ya las problemáticas y dificultades que conlleva que una Metrópoli en formación logre alcanzar las metas que la posicionen como un territorio sustentable, pero para tener claro cuáles son estas metas es importante basarse en estándares globales de medición, en este caso el Objetivo 11 de la Agenda 2030 para el Desarrollo Sostenible, si bien, tanto las ciudades como las zonas metropolitanas debieran tener una visión completa en lo que respeta a los Objetivos del Desarrollo Sustentable, es el punto 11 el que marca una pauta y una necesidad especifica de las Metrópolis para generar cambios.

De acuerdo con el Objetivo 11, las ciudades y áreas metropolitanas son centros neurálgicos del crecimiento económico, quienes contribuyen con el $60 \%$ del producto interno bruto mundial pero a su vez representan el $70 \%$ de las emisiones de carbono.

Es por ello que se plantean metas, que marcan la pauta para acercarse a la sustentabilidad de acuerdo a la Organización de las Naciones Unidas (CEPAL, N.U., 2019), las cuales responden al derecho a los servicios públicos, seguridad, movilidad digna y sustentable, espacios públicos de calidad, servicios públicos óptimos, áreas verdes, inclusión, impacto ambiental y patrimonio cultural.

Hemos decidido exponer, como objeto de estudio, un caso de crecimiento urbano y regional, local en la zona norte de América Latina, desde la lógica de la modernidad, viable económica y políticamente, pero sin considerar en toda su dimensión, las implicaciones medioambientales, en el contexto del desarrollo sustentable. Es el caso del tren interurbano Toluca-México que inició como gran proyecto federal (2012-2018), para dar respuesta a demandas de movilidad y transporte, pero que no fue concluido por diversos motivos. Entre ellos ausencia de previsión de gastos y elevación de costos. Aquí ha interesado analizar las consecuencias de la entrada en operación del sistema de transporte ferroviario, y la imperiosa necesidad de cooperación intermunicipal, desde las políticas públicas locales, en el contexto de las necesidades regionales específicas y tangibles, pero de evidente desarticulación. Ello queda de manifiesto cuando cada Gobierno municipal diseña y despliega sus planes de desarrollo, sin considerar del todo, una visión global como metrópoli, donde, 
por ejemplo, las estrategias de la Agenda 2030, como los Objetivos del Desarrollo Sostenible, aun siendo reconocidos, quedan al margen y no resultan prioritarios, lo que evidencia una ausencia de coordinación. Los planes de desarrollo de los ayuntamientos que conforman la ZMVT muestran una falta de coordinación, incluso en consonancia con las políticas del Gobierno del Estado de México. Aquí proponemos, en el contexto del paradigma del desarrollo sustentable, dadas las implicaciones en las esferas económica, social y natural, la imperiosa necesidad de una articulación, no sólo política, sino incluso educativa, en particular desde la educación superior, como es el caso de la MESRyM.

Desde dentro, porque hemos sido partícipes, de la administración municipal del Ayuntamiento de Toluca, se ha podido constatar, digamos la buena intención en la ejecución de acciones de índole sustentable, pero se observa ausencia de conocimiento, de convicción y de coordinación con otros entes de Gobierno municipal.

\section{CONCLUSIONES}

Sin lugar a dudas la implementación de medidas y acciones estratégicas en materia de sostenibilidad no es una tarea sencilla para ninguna ciudad o territorio, en el ámbito de administraciones y gobiernos locales, municipales. Generar cambios en pro de una perspectiva de ciudades sustentables, implica abordar desde diversas perspectivas, retos que involucran problemáticas de la ciudad, el territorio y la relación estrecha con la naturaleza como medio ambiente. En este caso que se analiza del Altiplano mexicano, como zona metropolitana en constante crecimiento, es posible identificar transformaciones en las infraestructuras por las diversas demandas, de comunicación y transporte, alojamiento y vivienda, seguridad y sanidad públicas. Las esferas de la sustentabilidad en cuanto a las dimensiones implicadas, naturaleza, sociedad y economía, posibilitan identificar la relación estrecha y compleja que establecen con el ser y devenir de las metrópolis. Aquí hemos analizado, aunque parcialmente, considerando sólo algunos aspectos que estimamos pertinentes en nuestro caso de estudio, en relación con la Agenda 2030 y el paradigma de la sustentabilidad.

Las ciudades complejas pueden comprenderse también en consideración a los problemas estructurales e, incluso históricos, como la variable demográfica, en crecimiento constante. Ante ello permanece vivo el ideal moral y político de aspirar a la mejor organización social, para afrontar y resolver las carencias y limitaciones de diversa índole, ante una realidad en constante cambio y transformación. En este esquema cabe a la perfección la noción de Responsabilidad, como un cuerpo teórico ético, para afrontar retos y asumir compromisos en las acciones, desde personales a empresariales, institucionales y de gobiernos, en sus distintos niveles. De ahí que se ha destacado la responsabilidad social para afrontar y hacer realidad ciudades sustentables.

No obstante lo anterior, la lucha de intereses, la desinformación, la ausencia de voluntad política pueden constituir factores que mermen los esfuerzos de quienes buscan generar cambios: culturales, económicos, políticos, estructurales. A casi una década de que venza el plazo para la implementación de los ODS, las transformaciones, modificaciones de hábitos y maneras de consumir y vivir, como cambios se ven lejanos; de ahí que las metas también se ven difíciles de alcanzar porque la visión y voluntad de los agentes que gobiernan y toman decisiones, parece ajena y distante de compromisos sustentables.

Por ello, se resalta la importancia de dos posibilidades que podrían favorecer y facilitar este proceso; por un lado, la necesidad de políticas públicas claras y responsables que regulen y sancionen los esfuerzos, tanto de las capacidades propias del estado, como de los actores empresariales y de la ciudadanía. Por otro lado, y quizás el más importante, la necesidad de que exista este proceso antes mencionado de ciudadanización, en donde los habitantes conozcan todos sus derechos y obligaciones, pero a su vez también se promueva la conciencia de la población, para destacar la correlación existente entre nuestro actuar como ciudadanos, tanto con los problemas de la ciudad como con las soluciones probables.

Esta conciencia de nuestra relación directa con las problemáticas metropolitanas permitiría que la Responsabilidad Social sea justamente eso, un entendimiento de nuestro compromiso hacia nuestra ciudad y territorio; hay también una corresponsabilidad con nuestros gobiernos y nuestro comportamiento como ciudadanos, ello debe conducirnos a acciones que respondan a un compromiso cívico, moral, democrático con nuestro medio ambiente. Dicho de otra manera, debemos asumir la responsabilidad de apropiarse de las problemáticas, como copartícipes de ellas, en tanto compartimos un mismo espacio, cultura e historia como comunidad viva, en relación imprescindible con la naturaleza. Pensar y actuar así es hacerlo responsable, sustentablemente. 


\section{REFERENCIAS}

Borja, J. (2008). "Los nuevos derechos ciudadanos", en HIC al, El derecho a la ciudad en el mundo, México: HIC AL

Cabrera Becerra, V. (2010). "Diagnóstico de competitividad del Municipio de Puebla, una aproximación a su complejidad". Puebla, Publicado por: Benemérita Universidad Autónoma de Puebla.

CEPAL, N. U. (2019). La Agenda 2030 y los Objetivos de Desarrollo Sostenible: una oportunidad para América Latina y el Caribe (LC/G.2681-P/Rev.3), Santiago. Recuperado de: https://repositorio.cepal.org/ handle/11362/40155

De Mattos, C. A. (1999). Teorías del crecimiento endógeno: lectura desde los territorios de la periferia. Brasil. Recuperado de: https://www.scielo.br/j/ea/a/65CP6fmZcZC9rL9yjZ9q4vx/?lang=es

Elkington, J. (2004). Enter the triple bottom line. Recuperado de: https://www.johnelkington.com/archive/ TBL-elkington-chapter.pdf

Estrada, E. M. R., Agüero, M. V. J., \& Cuenca, S. Q. (2013). Ciudades saludables: una perspectiva de la organización mundial de la salud y redes internacionales. Revista Legado de Arquitectura y Diseño, (13), 47-57.

García Galván M. (2019). El Tren Interurbano México-Toluca, un proyecto que atenta en contra del medio ambiente y los derechos humanos en La Marquesa. Revista de Geografía Espacios. Vol. 9 (17), 109- 134. Recuperado de: https://doi.org/10.25074/07197209.17.1225

Hancock T. (1993). "The Evolution, Impact and Significance of the Health Cities/Healthy Communities" Movement Journal of Public Health Policy volume 14, Palgrave Macmillan Journals, Recuperado de: https://doi.org/10.2307/3342823

Ingarden, R. (2001). Sobre la responsabilidad: sus fundamentos ónticos, Dorcas Caparrós editores.

Lefebvre, H. (1991). O direito a cidade, Sao Paulo: Editora Moraes

Morin E. (2012). Ethos y complejidad: hombre, naturaleza y vida. Citado en J. Loreto Salvador Benitez, ¿Hay lugar para la persona hoy? W. R. Darós (Coord.) UCEL, Rosario.

Organización de las Naciones Unidas O.N.U. (2010). Modelo de liderazgo en la sostenibilidad corporativa.

Santana Juárez, M. V., Rosales Estrada, E.M., Manzano Solís, L.R., Santana Castañeda, G., \& Pineda Jaimes, N. B. (2013). Las geotecnologías en la construcción de ciudades saludables: zona metropolitana de Toluca, México. Universidad Autónoma del Estado de México Facultad de Geografía Cerro de Coatepec s/n Ciudad Universitaria. Toluca, México, C.P. 50110. Recuperado de: http://ri.uaemex.mx/ handle/20.500.11799/58123

Serna M. G. (2006). Concepto de Responsabilidad Social Empresarial y su aceptación en las empresas Mexicanas. 2006, UNAM. 